\title{
CD99 marks malignant myeloid stem cells
}

Self-renewing cancer stem cells drive disease in acute myeloid leukaemia (AML) and myelodysplastic syndromes (MDS), but efforts to selectively target these cells have been hampered by a lack of specific markers. Now, Chung and colleagues have identified CD99 as a cell surface marker of disease stem cells in AML and MDS and show that a monoclonal antibody against CD99 has anti-leukaemic activity in xenograft mouse models.

AML and MDS are cancers of the haematopoietic system with shared molecular origins, and some patients with MDS go on to develop AML.

In the current study, the authors applied transcriptomics and flow cytometry to leukaemic blasts from patients with AML and to purified haematopoietic stem cells (HSCs) from patients with MDS. Compared with cord blood and healthy adult control HSCs, these cells showed significantly higher surface expression of CD99.

Next, Chung et al. focused on $\mathrm{CD} 34^{+} \mathrm{CD} 38^{-}$haematopoietic stem and progenitor cells (HSPCs) that were isolated from patients with AML, as this cell fraction is thought to be enriched for normal and leukaemic stem cells. CD99cells from this population, when transplanted into irradiated mice, formed lymphomyeloid engraftments that lacked leukaemia-associated mutations, which is consistent with the function of normal HSCs. By contrast, transplantation of HSPCs bearing CD99 resulted in engraftment of a lethal myeloid leukaemia. These studies demonstrate the utility of CD99 for prospective isolation of leukaemic stem cells.

The researchers also showed that the level of CD99 expression by leukaemic blasts correlated with stem cell function. Following transplantation into irradiated mice, leukaemic blasts with the highest (top 10\%) expression level of CD99 showed leukaemia-initiating cell activity, whereas cells with the lowest (bottom 10\%) CD99 levels did not include functional leukaemic stem cells. RNA sequencing of CD99 $9_{\text {hi }}$ versus $\mathrm{CD} 99^{\text {low }}$-expressing blasts revealed distinct gene signatures, including enrichment for leukaemic stem cell-associated genes in CD99 overexpressing cells. Importantly, unlike previously reported markers, CD99 was able to distinguish leukaemic stem cells in CD34- AML.

In vitro, two anti-CD99 antibodies were found to be directly cytotoxic to AML and MDS stem cells from patient samples, at concentrations that had no detectable toxicity against human umbilical vein endothelial cells (healthy endothelial cells also express low-intermediate levels of CD99).

In mice that received transplants of leukaemic blasts, a single treatment with anti-CD99 antibodies after 2 weeks completely abolished AML engraftment. Moreover, in mice with established leukaemic engraftment in bone marrow and peripheral blood, treatment for 4 weeks significantly reduced the leukaemic burden in both compartments.

Last, the researchers showed in AML cell lines that blocking CD99 triggers the activation of SRC family kinase (SFK) and thereby promotes oncogenic stress and cell death in myeloid leukaemias.

Given the key role of cancer stem cells in treatment resistance and relapse, the current study provides an important target to potentially improve outcomes in AML and MDS.

Katie Kingwell

ORIGINAL ARTICLE Chung, S. S. et al. CD99 is a therapeutic target on disease stem cells in myeloid malignancies.Sci. Transl Med. http://dx.doi. org/10.1126/scitranslmed.aaj2025 (2017) 